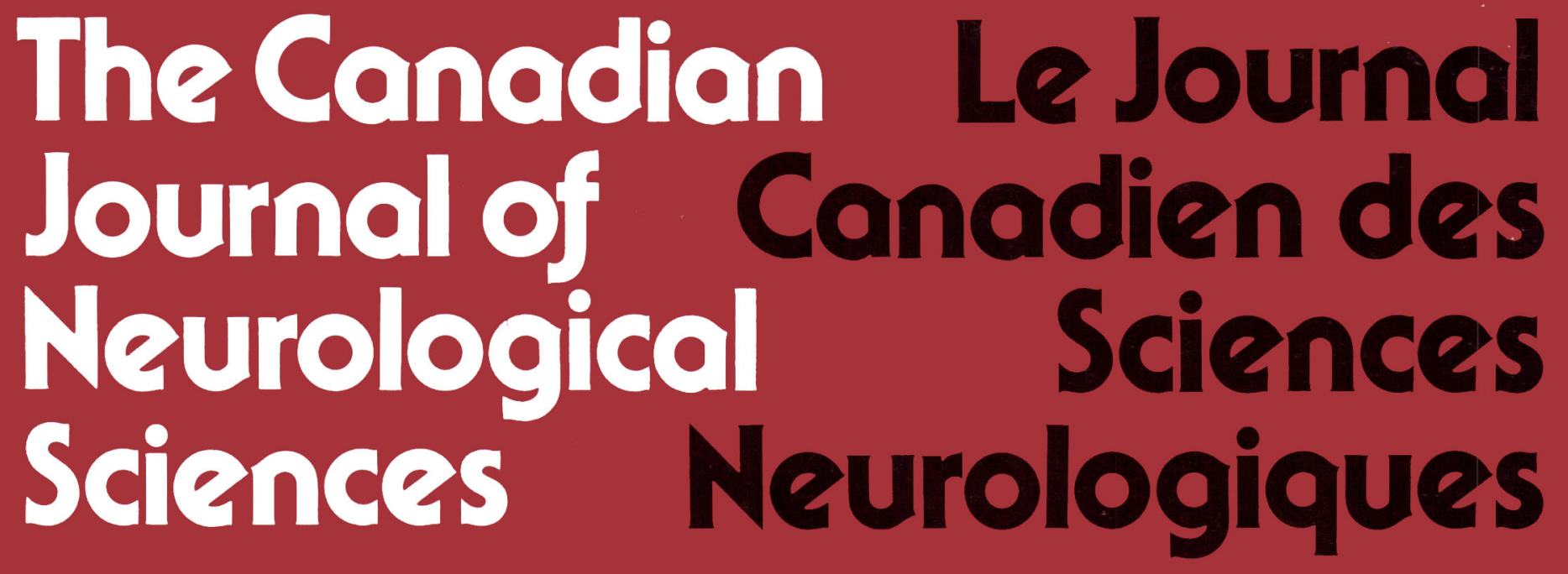

\title{
SUPPLEMENT
}

\section{ABSTRACTS OF THE Vth INTERNATIONAL SYMPOSIUM ON CEREBRAL VASOSPASM}

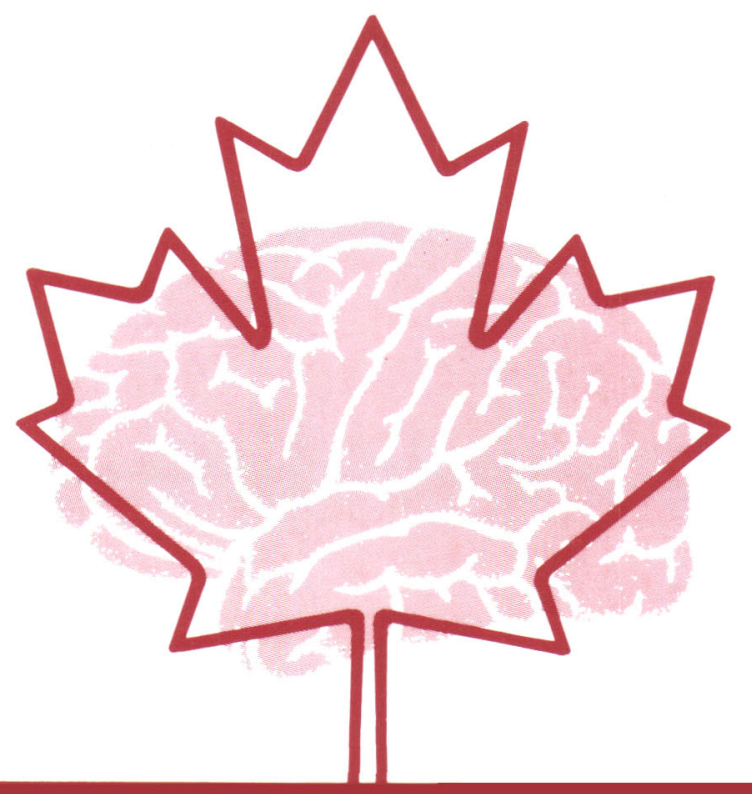

May $18-21,1993$

Edmonton and Jasper, Alberta

The Official Journal of

The Canadian Neurological Society

The Canadian Neurosurgical Society

The Canadian Society of Clinical Neurophysiologists

The Canadian Association for Child Nẹurology 


\section{PRESIDENT}

Bryce K.A. Weir

Edmonton

HONORED GUEST

Neal F. Kassell

Charlottesville

SECRETARY-TREASURER

J. Max Findlay

Edmonton

ADVISORY COMMITTEE

John Alksne

San Diego

T.B. Bolton

London, U.K.

John Bevan

Burlington

Douglas Chyatte

Chicago

N. Dorsch

Westmead

Namio Kodama

Fukushima

Marc Mayberg

Seattle

S. Moncada

Kent

H. Nornes

Oslo

Takashi Ohmoto

Okayama

Tomio Ohta

Fukushima

John W. Peterson

Boston

Kenneth Petruk

Edmonton

John Pickard

Cambridge

Isamu Saito

Tokyo

Tomio Sasaki

Tokyo

R.P. Sengupta

Newcastle Upon Tyne

Masato Shibuya

Nagoya

Robert R. Smith

Jackson

Thoralf Sundt

Rochester

R.H. Wilkins

Durham

Akira Yamaura

Chiba-shi

Takashi Yoshimoto

Sendai

Nicholas Zervas

Boston

Yuri Zubkov

St. Petersburg

\section{Vth International Conference}

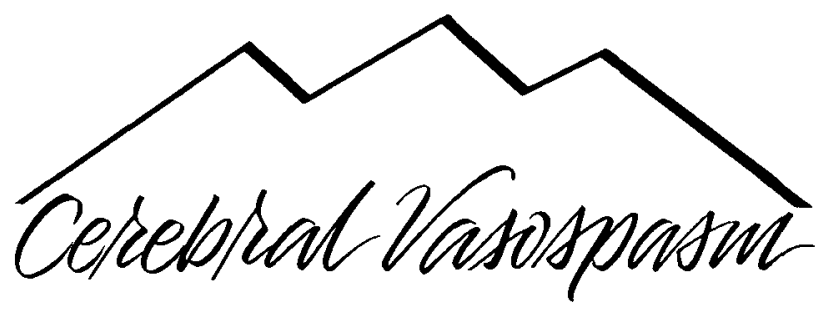

AN INTERNATIONAL SYMPOSIUM

$19 \div 93$

May $18-21,1993$

Edmonton and Jasper, Canada

\section{MEETING SECRETARIAT}

c/o Canadian Congress of Neurological Sciences 810, 906 - 12 Avenue S.W.

Calgary, AB, Canada T2R $1 \mathrm{~K} 7$

Tel: (403) 229-9544

Fax: (403) 229-1661 
Editor/Rédacteur en chef

James A. Sharpe Toronto, ON

\section{Associate Editors/Rédacteurs associés}

Laurence E. Becker Toronto, ON

John P. Girvin London, ON

Terry W. Picton Ottawa, ON

\section{Past Editors}

Robert G. Lee Calgary, $A B$

Robert T. Ross (founding editor)

Winnipeg, $M B$

\section{Editorial Board/Conseil Scientifique}

Warren Blume London, ON

Jean-Pierre Bouchard Québec, $P Q$

Donald Calne Vancouver, $B C$

Peter Camfield Halifax, NS

Pierre Duquette Montréal, $P Q$

Peter J. Dyck Rochester, MN, USA

George C. Ebers London, ON

Serge Gauthier Montréal, $P Q$

Julian T. Hoff Ann Arbor, MI, USA

Peter Humphreys Ottawa, ON

Richard Leblanc Montréal, $P Q$

George Karpati Montréal, $P Q$

Patrick McGeer Vancouver, $B C$

Ali H. Rajput Saskatoon, $S K$

William Pryse-Phillips St. Johns, NF

Richard ). Riopelle Kingston, $O N$

Richard Stein Edmonton, $A B$

John Stewart Montréal, $P Q$

Garnette R. Sutherland Winnipeg, $M B$

Charles Tator Toronto, ON
Book Review Editor/

Rédacteur de critiques de livres

Mary Anne Lee Calgary, $A B$

News Editor/Rédacteur (nouvelles)

John Norris Toronto, ON

Managing Editor/Administratrice adjointe

Sally A. Gregg Calgary, $A B$

Publications Committee/Comité de Rédaction

$\begin{array}{ll}\text { William F. Brown } & \text { Boston, MA USA } \\ \text { Donald Brunet } & \text { Kingston, ON } \\ \text { Gary Ferguson } & \text { London, ON } \\ \text { Frances Booth } & \text { Winnipeg, MB }\end{array}$

The Official Journal of:/La Revue Officielle de:

The Canadian Neurological Society

La Société Canadienne de Neurologie

President/Président - C.W. McCormick

Secretary-Treasurer/Secrétaire-Trésorier - O. Suchowersky

The Canadian Neurosurgical Society

La Société Canadienne de Neurochirurgie

President/Président - H.J. Hoffman

Secretary-Treasurer/Secrétaire-Trésorier - Renn Holness

The Canadian Society of Clinical Neurophysiologists

La Société Canadienne de Neurophysiologie Clinique

President/Président - Bryan Young

Secretary-Treasurer/Secrétaire-Trésorier - Michael jones

The Canadian Association for Child Neurology

L'Association Canadienne de Neurologie Pédiatrique

President/Président — Shashi Seshia

Secretary-Treasurer/Secrétaire-Trésorier — William Logan

The permanent secretariat for the 4 societies and the Canadian Congress of Neurological Sciences is at/

Le secrétariat des 4 associations et du Congrès Canadien des Sciences Neurologiques est situe en permanence à:

$810,906-12$ Avenue S.W., Calgary, AB Canada T2R 1K7

The Canadian Journal of Neurological Sciences is published quarterly. The annual subscription rate is $\$ 60$ for members; $\$ 70$ for non-members in Canada; $\$ 80$ for USA and elsewhere. Residents, Interns, Pre- and Post-Doctoral Students $\$ 30$ per annum (members); $\$ 40$ per annurn (non-members). Single copies $\$ 18$ each plus postage and handling. All manuscripts and communications should be sent to: Canadian lournal of Neurological Sciences, P.O. Box 4220, Station C, Calgary, AB Canada T2T 5N1. Courier to: 8th Floor, 906 - 12 th Avenue S.W., Calgary, AB Canada T2R 1 K7. Telephone (403) 229-9575.

COPYRIGHTC 1993 by THE CANADIAN JOURNAL OF NEUROLOGICAL. SCIENCES INC. No part of this journal may be reproduced in any form without the prior permission of The Canadian Journal of Neurological Sciences. Mailed under Publications Mail registration number 3307. Postage paid at Calgary, Alberta. This journal is indexed by Index Medicus, Excerpta Medica and Current Contents - Clinical Practice and Life Sciences.

Le Journal Canadien des Sciences Neurologiques est publié trimestriellement. L'abonnement annuel est de $60 \$$ pour les membres; $70 \$$ pour les non-membres au Canada; $80 \$$ pour les Etats Unis et ailleurs. Internes, résidents, fellows pré et post doctoral: $30 \$$ par année (membres); $40 \$$ par année (non-membres). Copie sinple: $18 \$$ plus affranchissement et manutention. Toutes les communications et les manuscrits doivent être adressés à Journal Canadien des Sciences Neurologiques, P.O. Box 4220, Station C, Calgary, AB Canada T2T 5N1. Par courrier: 8th Floor, 906-12th Avenue S.W., Calgary, AB Canada T2R 1K7. Téléphone (403) 229-9575.

DROITS D'AUTEURO 1993: THE CANADIAN IOURNAL OF NEUROLOGICAL SCIENCES INC. Aucune partie de ce lournal ne peut être reproduite, sous quelque forme que ce soil, sans la l'authorisation du Journal Canadien des Sciences Neurologiques. Posté sous permis de poste-publications no 3307 . Port payé à Calgary, Alberta. Le lournal est cité et indexé dans Index Medicus, Excerpla Medica et Current Contents - Clinical Practice et Life Sciences.

Advertising representative/Représentant de publicité Sally Gregg, Canadian Journal of Neurological Sciences

810, 906 - 12 Ave. S.W., Calgary, AB Canada T2R 1K7 - (403)-229-9575

Printer/Imprimeur MCAra Printing Limited, 105, 2507 - 12th Street N.E., Calgary, Alberta T2E 7L5

ISSN 0317 - 1671 